\title{
Interpolation in structure from motion
}

\author{
ASAD SAIDPOUR, MYRON L. BRAUNSTEIN, and DONALD D. HOFFMAN \\ University of California, Irvine, California
}

\begin{abstract}
We investigated surface interpolation in displays of structure from motion (SFM). To do so, we introduced a new method for measuring surface perception in dynamic displays-the SFM probe. An SFM probe is a dot that moves rigidly with the dots on a simulated surface, and whose distance from that surface can be adjusted with a joystick or similar control. The displays we studied were random-dot cylinders containing a vertical strip devoid of feature points (the gap). Subjects adjusted an SFM probe, presented in the gap, until the probe dot appeared to be on the surface. Variability in probe-dot placement decreased with increasing texture density on the cylinder and increased with increasing gap width. Subjects showed a consistent bias to place the probe dot outside the cylinder. This bias increased with increasing texture density for the SFM displays. (The opposite bias was found in a static two-dimensional interpolation task with an arc whose curvature matched that of the cylinder: Subjects placed the probe dot inside the arc.) This outside bias is inconsistent with several theoretical approaches to surface interpolation.
\end{abstract}

In a typical structure-from-motion (SFM) display, several dots move about on a computer-driven display. The dots move, of course, in the plane of the display. However, for certain motions of the dots, subjects report that the dots appear to move in three dimensions, not in the plane of the display (Braunstein, 1962, 1976; Green, 1961; Ullman, 1979; see also Wallach \& O'Connell, 1953). Moreover, subjects often report that the dots move together rigidly. In some cases, primarily when there are few dots, subjects may report that the dots appear to be connected by imaginary lines. In other cases, primarily when there are many dots, subjects may report seeing a surface in three dimensions (such as a sphere, a cylinder, or some other shape-see, e.g., Braunstein \& Andersen, 1984), with the dots appearing to be little lights attached to the surface. The impression of a surface can be quite compelling, particularly if the display is composed of light dots against a dark background and the display is viewed monocularly in a darkened room. The surface is, nonetheless, not physically present in the display, suggesting that subjects interpolate a "subjective surface" between visible feature points in an SFM display.

There have been many psychophysical studies of the perception of SFM. Most have investigated the minimal conditions (i.e., the minimal numbers of points or views) for structure to be perceived, and how the perceived structure varies as one varies the numbers of points and views (Braunstein, Hoffman, \& Pollick, 1990; Braunstein, Hoffman, Shapiro, Andersen, \& Bennett, 1987; Doner, Lappin,

This research was supported by Office of Naval Research Contract NOO014-88-K-0354 and National Science Foundation Grant BNS-8819565. We would like to thank Bruce Bennett and Jeffrey Liter for helpful discussions and George Andersen for comments on an earlier draft of this paper. Correspondence should be addressed to Myron L. Braunstein, Department of Cognitive Sciences, University of California, Irvine, CA 92717 (e-mail: mlbrauns@orion.oac.uci.edu or mlbrauns@uci.bitnet).
\& Perfetto, 1984; Dosher, Landy, \& Sperling, 1989; Lappin, Doner, \& Kottas, 1980; Petersik, 1987; Todd, Akerstrom, Reichel, \& Hayes, 1988; Treue, Husain, \& Andersen, 1991). No psychophysical studies have investigated the interpolation of surfaces in regions of a display devoid of feature points. To help fill this gap, the experiments described here provide quantitative data on surface interpolation for a particular combination of structure and motion: a cylinder rotating about a vertical axis.

Although surface interpolation has not heretofore been studied in the context of SFM, it has been studied in other contexts, such as static monocular figures and stereograms (see Figure 1). Stevens and Brookes (1987) showed subjects line drawings similar to that in Figure 1a, and they had them judge the slant of the normal to the subjective surface (i.e., the angle between the normal and the line of sight) at various locations on the surface. Such judgments were in good agreement with predictions that were based on the following hypothesis: that human vision synthesizes a subjective surface for which the given curves are lines of principal curvature (this hypothesis is discussed in Stevens, 1981, 1983, 1986). Stevens and Brookes (1987) then had subjects fixate a central point of the figure and judge the apparent depths of various other points on the subjective surface, using a dot presented stereoscopically as a depth probe. The results suggest that subjects took the fixation point to lie at the same absolute distance as the stereo horopter (locus of zero disparity), and that subjects integrated the local surface orientation to obtain estimates of the depth at other points. This integration process was more robust for figures generated by perspective projection rather than orthographic (parallel) projection.

In the case of stereograms, it has long been known that very sparse stereograms, with dot densities as low as $1 \%$, lead to strong impressions of subjective surfaces (Julesz, 1971; Julesz \& Frisby, 1975). Uttal, Davis, Welke, and Kakarala (1988) showed subjects stereograms, similar to 


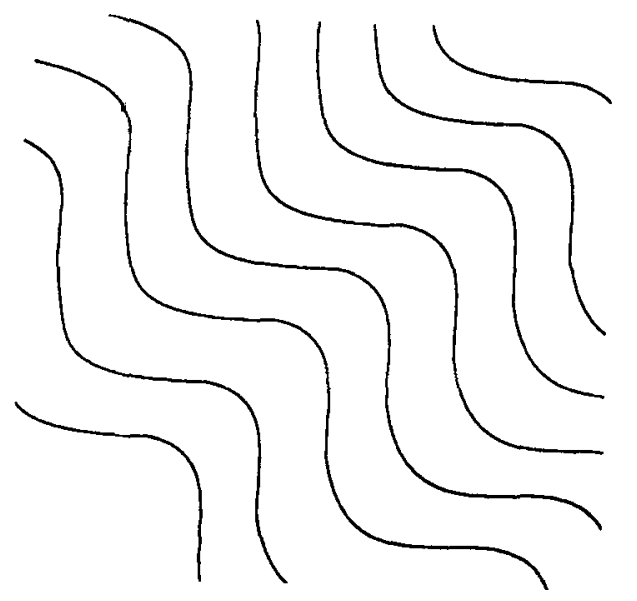

a

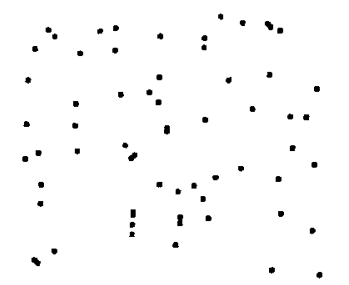

Figure 1. Examples of (a) a static monocular figure of the type used by Stevens and Brookes (1987) to study surface interpolation and (b) a stereogram of the type used by Uttal, Davis, Welke, and Kakarala (1988).

that in Figure 1b, depicting eight different quadric and cubic surfaces. Each time a subject was shown a stereogram, the subject decided which of the eight surfaces it represented. The data indicate that subjects can perform this task at better than chance levels with as few as four points, and that performance improved as more points were added, until, with just 10 visible points, subjects were correct on more than $90 \%$ of their judgments. This led Uttal et al. to suggest that visual reconstruction of surfaces from stereo data can proceed with very few points, certainly less than 10 and perhaps as few as 4 .

Bülthoff and Mallot (1987) constructed a stereogram in which a black annulus with crossed disparity was shown superimposed on a larger gray disk that had zero disparity. Because the interior of the annulus was featureless, as was the visible interior of the disk, the only source of disparity information was the edge of the annulus and the edge of the disk. Human vision apparently interpolates the depth between the edge of the disk and the outer edge of the annulus, leading to the perception of a truncated cone. Würger and Landy (1989) studied such interpolation in more detail, showing subjects a stereogram in which a rectangle with a gray featureless interior was superimposed on a larger background of random dots. The left edge of the rectangle had zero disparity, as did also the background. The right edge of the rectangle had nonzero disparity (either $20^{\prime}$ or $30^{\prime}$ of arc), leading to the perception of a plane attached to the background at the left side and slanting toward the viewer at the right side. Since the interior of the rectangle was featureless, disparity information was available only at the edges. By means of a stereo probe dot, subjects indicated what depths they saw at various points within the rectangle. The data suggest that subjects do not see a simple linear interpolation between the edges of the rectangle, but see instead a curved surface that sags, much as though one draped a wet cloth on a rectangular wire frame but did not pull the cloth completely taut over the frame.

An experiment by Brookes and Stevens (1989) suggests, however, that care must be taken in interpreting the data obtained by stereo probes: there are conditions in which the apparent depth of a probe dot is not solely, or even primarily, a function of the disparity of the dot. Indeed, one can construct stereograms in which the depth order of two probe dots is mistakenly reversed-for example, in which the disparities of dots $A$ and $B$ are such that $A$ should appear closer than $B$, but for which subjects report the opposite order. In the example given by Brookes and Stevens, the stereogram gives rise to the percept of a subjective surface (a staircase), and this surface seems to "pull" the probe dots, overriding their disparities and leading to an incorrect interpretation of their depths.

Grimson (1981) presented another interesting demonstration of subjective surfaces in stereograms. He showed a random-dot stereogram of $10 \%$ density, which, when fused, looked like a half cylinder lying on a flat background (see Figure 2). The interesting twist was that the density of dots reduced gradually to zero in the center of the cylinder, yet the perception of a surface was quite strong even in the resulting gap. Grimson suggested three possible surfaces that might be interpolated into this gap: (a) a single planar surface connecting the edges of the gap, (b) a cylinder, or (c) a dihedral surface obtained by extending the tangent planes of the cylinder at the two edges of the gap and allowing them to intersect in a line of dis-
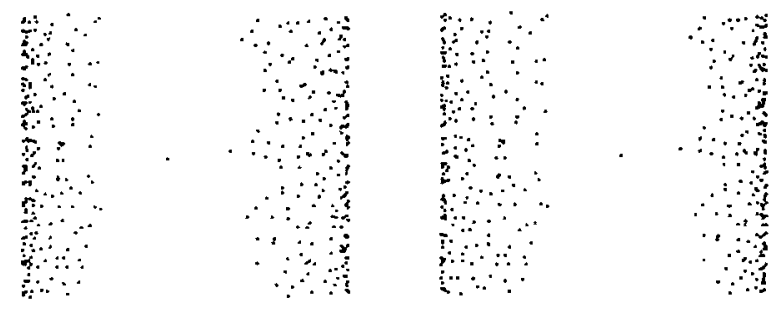

Figure 2. A stereogram of a half cylinder with a central gap, similar to that used by Grimson (1981) to demonstrate surface interpolation. 


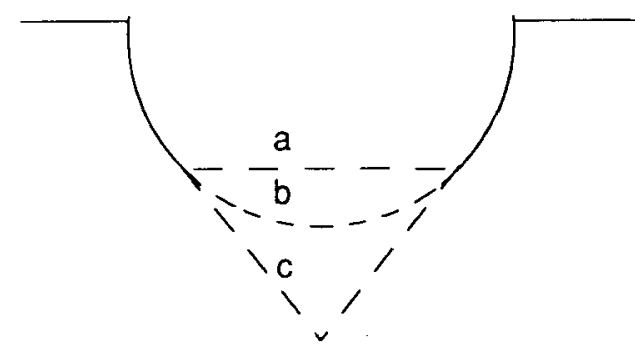

Figure 3. Three surfaces that might be interpolated through a gap in a cylinder (see text).

continuity (see Figure 3). If a stereo probe dot is placed in the gap at a depth corresponding to Possibility a, it appears to lie behind the subjective surface. If the probe is placed at a depth corresponding to Possibility c, it appears to lie in front of the subjective surface. When placed at a depth corresponding to Possibility b, it appears to lie approximately on the surface. We mention Grimson's demonstration in some detail, because it provided the point of departure for the present experiments done with SFM. In these experiments, as we will discuss in more detail shortly, subjects were shown, by means of SFM, half cylinders that had a central gap. Into this gap we introduced an SFM probe dot, which subjects could adjust in depth, using a joystick, until the dot lay on the subjective surface that appeared to pass through the gap. The probe dot was not presented in stereo, although the use of such a probe could cast light on the integration of depth from stereo and SFM. Rather, the apparent depth of the probe was due entirely to its motion-hence it was an SFM probe. By allowing subjects to adjust the depth of this probe interactively, we could obtain quantitative data about the subjective surfaces that they saw in certain displays of SFM.

Our ultimate objective in determining how subjects place an SFM probe dot in a region devoid of feature points is to constrain possible theories. So before proceeding to a detailed description of our experiments, we will review briefly some of the developments in the mathematical theory of interpolation or, more generally, of approximation. In these approaches, we begin with a finite collection of points or curves in space, which, we assume, all lie on some surface that is otherwise not visible. We have, in effect, a sparse sample of the surface. Our goal is to recover a description of the entire surface, even in regions between the given samples. Clearly this problem is underconstrained: we could easily generate many different surfaces that pass through (or near) all the given features but that behave quite differently everywhere else. This suggests that we need to employ some constraint to pick out one "good" surface from among the possibilities. And indeed, the theoretical work on surface interpolation has been largely a series of proposals about what constraint to use.
Such a proposal is usually couched in the form of a functional-a mathematical function that takes large values for "bad" surfaces and smaller values for "better" surfaces. The functional typically embodies some intuition, such as that the best surface is that surface which, of all surfaces passing through (or near) the given features, is the smoothest. One proposal is the "quadratic variation" functional (Brady \& Horn, 1981; Grimson, 1981). If a surface $S$ is described by the set of points

$$
S=\{(x, y, z) \mid z=f(x, y)\}
$$

for some fixed function $f: \mathbf{R}^{2} \rightarrow \mathbf{R}$, then the quadratic variation functional $\Theta$ is given by

$$
\theta(f)=\left\{\iint\left(f_{x x}^{2}+2 f_{x y}^{2}+f_{y y}^{1}\right) d x d y\right\}^{1 / 2},
$$

where the subscripts indicate partial derivatives. Intuitively, this functional measures the curvature of the interpolating surface, and minimizing this functional gives a surface of minimal curvature-that is, a smoothest surface. One can think of the interpolating surface as the thin plate of least energy passing through the given points. There are several nice features of this functional: it is guaranteed to pick out a unique best surface (assuming there are at least four noncoplanar feature points in the image), it is circularly symmetric (so that rotating the image still leads to the same answer), and it is readily implemented. It is superior, in the view of Brady and Horn (1981) and Grimson (1981), to a similar functional, the "square Laplacian," which is given by

$$
\Theta(f)=\left\{\iint\left(\nabla^{2} f\right)^{2} d x d y\right\}^{1 / 2},
$$

because it requires fewer points to obtain a unique best surface, and because it gives more natural results at the boundaries of surfaces (i.e., where $f[x, y]$ goes to zero). However, as is well known to Brady and Horn (1981) and Grimson (1981), it has one primary deficiency: it smooths over discontinuities in the surface-depth discontinuities, such as the vertical drops in a staircase from one step to another, and orientation discontinuities, such as the peak of a roof. Where human vision may see a sharp discontinuity, both the quadratic variation and square Laplacian functionals interpolate a smooth surface, thereby eliminating the discontinuity.

Fixing this problem is a primary motivation of subsequent proposals. A three-stage approach was proposed by McLauchlan, Zisserman, and Blake (1987). One first interpolates a surface, then runs an edge-finding process to locate surface discontinuities, and finally reruns the interpolation process with the discontinuities marked. Another approach uses a functional that incorporates a term allowing discontinuities (Blake, 1984; Blake \& Zisserman, 1986, 1987; Marroquin, 1985; Terzopoulos, 1984, 1986). With discontinuities allowed in some regions, a smoother surface can be constructed in the remaining regions. This approach can be thought of as approximating the given features by using a thin plate or membrane that can bend and, if the stress of bending becomes too great, 
can also break. How great is too great? A principled answer to this question has not been forthcoming, so an arbitrary threshold is usually set, which is a drawback of this approach. This drawback can be overcome, however, by an approach that allows the thin plate not only to bend, as before, but also to compress and expand (Weiss, 1990). In regions where the given features have a sharp variation in depth, the plate can compress to give a better approximation. This gives a principled way to manage discontinuities in the process of surface reconstruction.

These developments are all quite recent, and no doubt there are more to come. For instance, no theory yet specifies when surface reconstruction should not be done. If we place a few dozen dots at random within the volume of a sphere and show these dots to subjects, either in stereo or in SFM, the subjects may report seeing no subjective surfaces at all, just dots filling a volume. A complete theoretical understanding of the human visual reconstruction of surfaces will have to account for the conditions in which subjective surfaces fail to appear, not just the shape of subjective surfaces when they do happen to appear. By analogy, theories of SFM (e.g., Ullman, 1979) specify conditions in which three-dimensional (3-D) structures are not expected to appear (e.g., by noting that in these conditions there are no rigid interpretations), not just descriptions of the shapes of 3-D structures that are recovered by the theory.

To relate theoretical accounts of interpolation to human perception, we need to know how accurately and reliably subjects can interpolate a surface between visible feature points. We conducted a series of four experiments by using displays of SFM in which subjects placed a probe dot on a simulated cylinder in a region devoid of feature points. The first experiment examined the effects of gap size, texture density, and cylinder radius on probe-dot placement. The second experiment was a partial replication of the first, except that the initial texture density was uniform in the two-dimensional (2-D) projection rather than uniform on the simulated 3-D surface. The third and fourth experiments examined the density and gap effects more closely, especially with respect to the precision of interpolation. In a fifth experiment, we introduced a 2-D interpolation task for comparison with the 3-D task.

\section{EXPERIMENT 1}

\section{Method}

Subjects. The subjects were 6 undergraduate students, who were paid for their participation. Acuity of at least 20/40 (Snellen eye chart) was required in the eye used throughout the experiment. The subjects had no knowledge of the purposes of the experiment. Four additional subjects participated in the experiment, but their data was excluded from the analysis because they failed a screening criterion. The criterion was that the probe dot should not be placed outside the surface by more than two radii on more than one trial. The criterion was based on preliminary observations that such extreme responses indicated a failure to understand the task.

Stimuli. The stimulus displays consisted of orthographic projections of dots on the surface of opaque cylinders oscillating $\pm 19^{\circ}$ around a vertical axis. ${ }^{1}$ Dots were positioned on the surface of the cylinder in the following manner. The surface of each cylinder was divided into a number of equal-area cells, with the number of cells determined by the density level (see below). One dot was placed randomly within the boundaries of each cell with a probability of 1, except for cells in the area of the gap. For cells in a vertical strip in the center of the gap, the probability of dot placement was 0 . For cells in vertical strips on either side of the central strip, and equal in width to half the central strip (in degrees of arc), the probability of dot placement increased linearly from 0 to 1 (see Figure 4). This ramp function was used to prevent sharp virtual contours around the gap, possibly resulting in the perception that an object was occluding the cylinder (see Grimson, 1981, for a similar precaution in the case of stereo interpolation). The gap widths reported in this paper were measured between the midpoints of the strips in which the dot-placement probabilities increased linearly. Specifically, the gap width was defined as the angle formed in a horizontal cross-section of the cylinder by connecting those midpoints to the center of the cylinder.

A single dot, the probe dot, was placed in the center of the gap (see Figure 4). Its vertical position was varied randomly within the middle $40 \%$ of the height of the cylinder, and its simulated position in depth was varied randomly between 50 and 200 pixels from the surface of the cylinder in either direction. ${ }^{2}$ To prevent the dot from moving outside the boundaries of the projected cylinder or disappearing from the display, its range of adjustment was limited to between 98 and 1638 pixels from the origin (center of the cylinder). The probe dot rotated in phase with the cylinder.

We define the position of the cylinder for which the gap and probe dot are centered in the image as the $0^{\circ}$ position. The cylinder started randomly at either $+19^{\circ}$ or $-19^{\circ}$ and accelerated sinusoidally from $0 \% \mathrm{sec}$, reaching its maximum speed of $15.35 \% \mathrm{sec}$ at the $0^{\circ}$ posi-

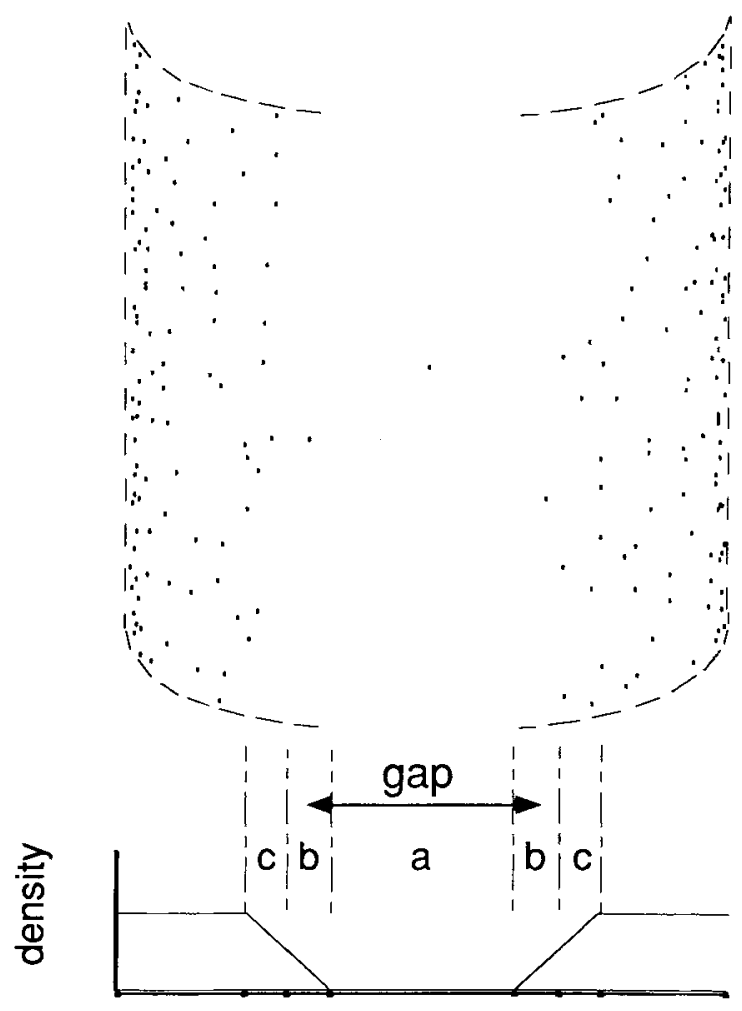

Figure 4. A random-dot display simulating a cylinder with a gap in its center and a probe dot in the gap (top), and the density profile for three regions of the cylinder (bottom). 
tion in $1.24 \mathrm{sec}$. It then decelerated from $15.35 \% \mathrm{sec}$ to $0 \% \mathrm{sec}$ in $1.24 \mathrm{sec}$, reversed direction, and continued to oscillate until the subject responded. The sinusoidal acceleration and deceleration were used to avoid sudden changes of direction. The edges of the cylinder were clipped at $Z=21$ pixels (to avoid the concentration of projected texture at the edges that would occur if the clipping were at $Z=0$ ).

Destgn. We examined three independent variables: cylinder radius, texture density, and gap size. The cylinder radius was 500 or 640 pixels. The texture-density levels were 0.13 dots $/ \mathrm{cm}^{2}, 0.21 \mathrm{dots} / \mathrm{cm}^{2}$, $0.53 \mathrm{dots} / \mathrm{cm}^{2}, 1.00 \mathrm{dots} / \mathrm{cm}^{2}$, and $1.39 \mathrm{dots} / \mathrm{cm}^{2}$ for the smaller radius, and $0.13 \mathrm{dots} / \mathrm{cm}^{2}, 0.25 \mathrm{dots} / \mathrm{cm}^{2}, 0.50 \mathrm{dots} / \mathrm{cm}^{2}, 0.99 \mathrm{dots} / \mathrm{cm}^{2}$, and 1.45 dots $/ \mathrm{cm}^{2}$ for the larger radius. (The density variations resulted from dividing cylinders with different dimensions into discrete numbers of cells.) The gap width was either $29^{\circ}$ or $54^{\circ}$. The levels of these independent variables were selected on the basis of preliminary experiments. Each subject responded four times to each of the 20 conditions.

Apparatus. The displays were presented on an IMI 455 vector graphics display system with $4096 \times 4096$ resolution. The dots were $1 \mathrm{~mm}$ wide $\times 2 \mathrm{~mm}$ high $\left(3.7^{\prime} \times 7.5^{\prime}\right.$ of arc). Subjects viewed the display monocularly in a darkened room from a distance of $91 \mathrm{~cm}$ through a tube arrangement that limited the field of view to an area within the borders of the display scope. A 0.5 neutral-density filter was inserted in the tube to remove any apparent traces on the CRT. The visual angles subtended by the cylinders (both height and width) were $5.47^{\circ}$ and $6.99^{\circ}$ for the small and large radius cylinders, respectively. A 570 -pixel-radius cylinder $\left(6.23^{\circ}\right)$ was used in the practice trials. The visual angle subtended by the projected gap varied with the radius of the cylinder and the angle of rotation. For gap widths of $29^{\circ}$ and $54^{\circ}$, the visual angles at $0^{\circ}$ rotation were $1.37^{\circ}$ and $2.48^{\circ}$ for the 500 -pixel-radius cylinder, $1.56^{\circ}$ and $2.83^{\circ}$ for the 570 -pixel-radius cylinder, and $1.75^{\circ}$ and $3.18^{\circ}$ for the 640 pixel-radius cylinder.

The subject's response device was a force joystick with an adjacent illuminated button. When the button was pressed, the computer recorded the amplitude of the probe-dot oscillation, coded as a distance from the origin of the simulated cylinder along a radius of the cylinder.

Procedure. Each subject participated in two experimental sessions. Each session began with 10 practice trials followed by a random sequence of the 20 conditions, a 1 -min rest period, and another random sequence of the $\mathbf{2 0}$ conditions. The density levels and gap sizes for the practice trials were the same as in the experimental trials. The interstimulus interval was approximately $3 \mathrm{sec}$.

Subjects were instructed to "move the middle dot until it is on the surface of the object" by using the joystick, and to press the button when they were satisfied with their adjustment. They were given a chance to practice with the joystick, to move it forward (toward the monitor) or backward and to observe the resulting changes in the motion of the probe dot. Forward motion of the joystick moved the dot closer to the center of the cylinder, and backward motion of the joystick moved the dot away from the center of the cylinder. The object remained visible until the subject pressed the button. The room was darkened 2 min before the trials began. All subjects were run without feedback.

\section{Results}

Of 480 responses (6 subjects responding four times to 20 conditions), the probe dot was placed outside the cylinder on 363 trials and inside the cylinder on 117 trials. Of the trials on which the probe dot was placed outside the cylinder, it was placed between the cylinder surface and the intersection of the tangent planes (i.e., the planes tangent to the cylinder at the two edges of the gap) on 139 trials and beyond this intersection (farther outside the surface) on 224 trials. Of the trials on which the probe dot was placed inside the cylinder, the dot was placed between the surface and a plane connecting the edges of the gap on 79 trials and between that plane and the center of the cylinder on 38 trials. (See Figure 5.) An analysis of variance (ANOVA) for the five density levels, two gaps, and two radii was conducted with constant error (CE)-the signed distance between the probe dot and the simulated surface-as the dependent variable. The main effect of density was significant $[F(4,20)=4.89, p<.05$, $\left.\omega^{2}=.102\right]$. As texture density increased, subjects placed the probe dot farther away from the surface. A post hoc analysis (Tukey's honestly significant difference test) showed the CE at Density Level 5 to be significantly greater than the CEs at Density Levels 2 and 3. All other pairs were nonsignificant. There was also a significant main effect for radius $\left[F(1,5)=15.48, p<.05, \omega^{2}=\right.$ $.015]$. This effect occurred because the $C E$ was approximately proportional to the radius. The mean CEs for the 500 - and 640-pixel radii were 46.1 and 54.0, respectively. ${ }^{3}$ There was a significant interaction between density and gap $\left[F(4,20)=4.37, p<.05, \omega^{2}=.044\right]$ and a significant interaction between density and radius $[F(4,20)$ $\left.=3.38, p<.05, \omega^{2}=.018\right]$. The main effect of gap size was not significant $[F(1,5)=.320]$. There were no other significant interactions. All subjects reported seeing a 3-D object all the time and a cylinder most of the time.

Although the screening criterion described above eliminated subjects who responded more than once with the probe dot more than two radii from the surface, the mean CEs for some conditions were affected by a small number of extreme responses. In later experiments (beginning with Experiment 3), more stringent screening criteria were used that eliminated responses exceeding 1.5 radii outside the surface. To facilitate comparison of the CE data with the data from these later experiments, we reanalyzed the data from Experiment 1, eliminating data from 16 trials (of a total of 480) on which the probe dot was placed more than 1.5 radii outside the surface. In this new analysis, the main effect of density remained significant $[F(4,20)=$

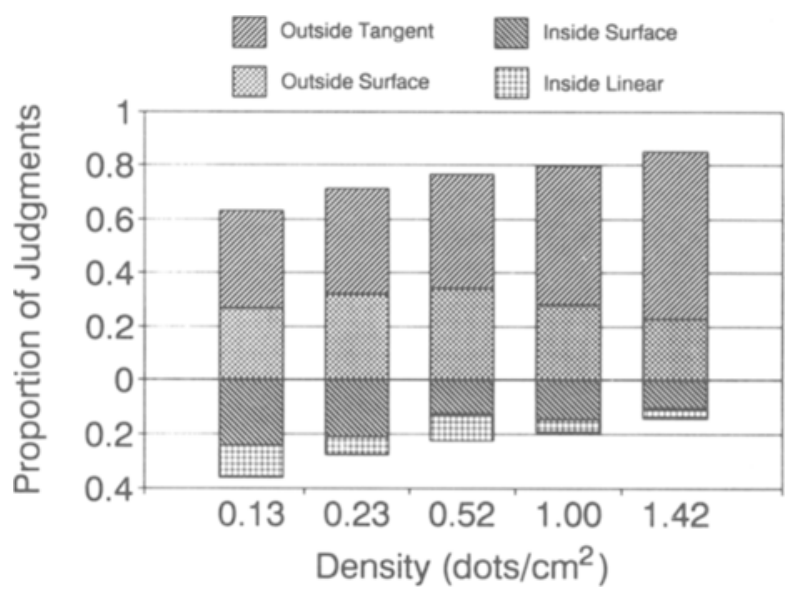

Figure 5. Probe-dot placement as a function of density in Experiment 1. 
4.65, $\left.p<.01, \omega^{2}=.052\right]$. The mean CEs for the five density levels were $27.3,26.1,29.9,44.7$, and 57.7. The mean CE for Density Level 5 was significantly different from the means for Levels 1, 2, and 3. No other main effects or interactions were significant.

\section{Discussion}

The principal finding of Experiment 1 was the bias to place the probe dot outside of the surface. This bias increased with increasing texture density. Figure 6 shows the relationship between probe-dot placement and the three locations of interest discussed earlier: the plane connecting the edges of the gap (the linear interpolation location), the surface, and the intersection of planes tangent to the surface at the edges of the gap. For the smaller gap, the mean probe-dot placement for all density levels was farther from the surface than the intersection of the tangent planes. For the larger gap, the mean probe-dot placement was between the surface and that intersection for all density levels. Although probe-dot placement did not consistently coincide with any of these three locations, it was farthest from the linear location and was closer to the tan- gent plane intersection than to the surface in most conditions. These results, however, do not indicate that probedot placement was influenced by the location of the tangent plane intersection. The change in relationship between the $\mathrm{CE}$ and the tangent plane intersection, from the smallgap condition to the large-gap condition, appears to be the result of the change in distance between the surface and that intersection that occurs as a geometric consequence of the change in gap width. This issue was addressed again in Experiment 4, in which five levels of gap width were studied.

\section{EXPERIMENT 2}

In Experiment 1, the dots were placed uniformly on the surface of the simulated 3-D cylinder. This provided a texture-density cue to curvature. As a control for this cue, we replicated most of the conditions from Experiment 1 with displays having (when the gap was centered in the image) uniform texture density in the image. This was only a partial control, because the projected density changed as the cylinder was rotated. Although we could
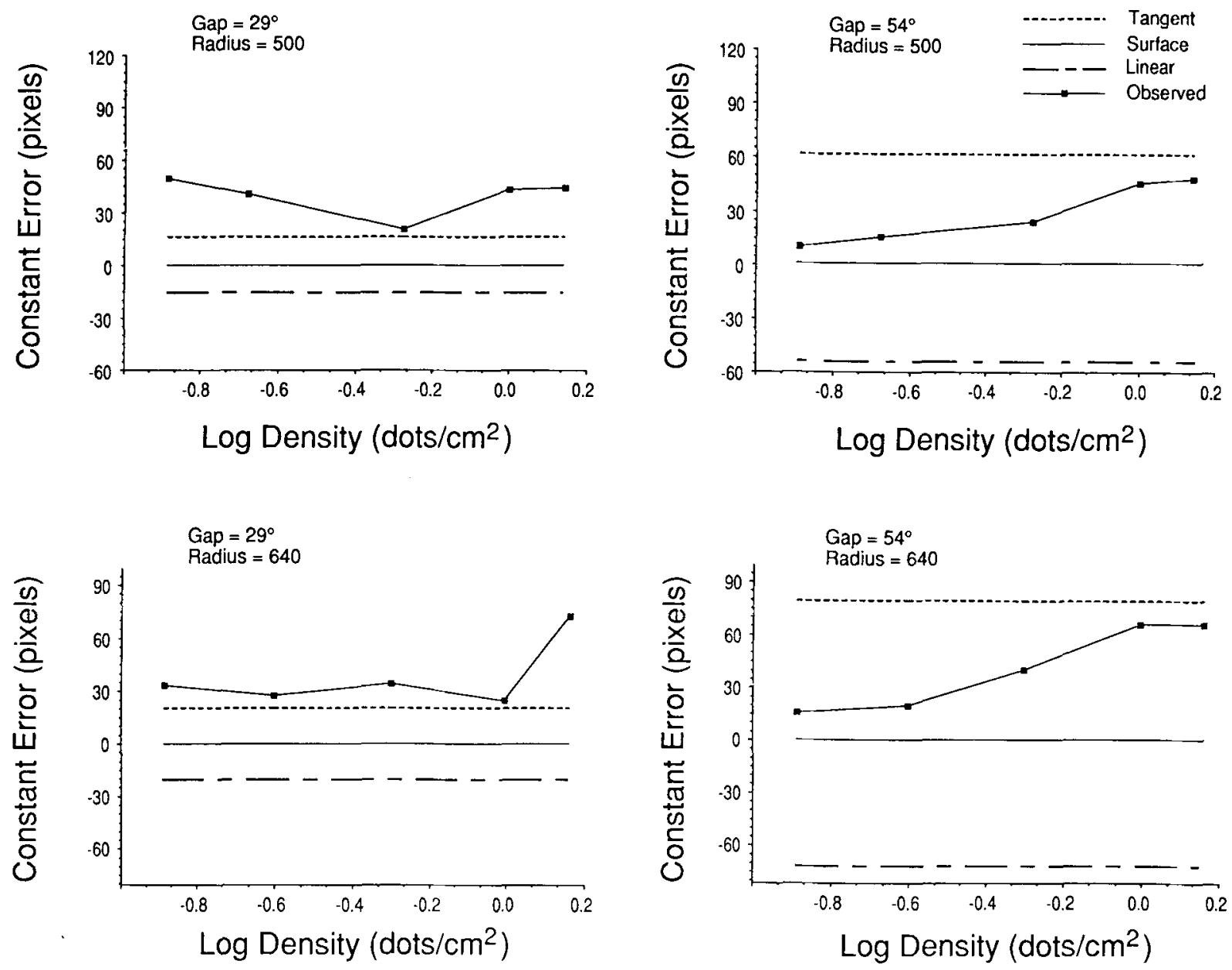

Figure 6. Constant error in probe-dot placement for four combinations of gap and radius. 
have maintained uniform texture density on each individual frame by adding and subtracting dots (Sperling, Landy, Dosher, \& Perkins, 1989), this would have produced scintillation in the image (Braunstein \& Todd, 1990). Because the cylinder rotated only $19^{\circ}$ in each direction, the changes in texture density due to rotation were small.

\section{Method}

The stimuli, apparatus, and procedure were identical to those in Experiment 1, except that when the gap was in the center of the screen, the texture was uniform in the 2-D image rather than uniform on the surface of the cylinder, as in Experiment 1. The same 6 subjects participated in Experiment 2.

We used the same gap and radius conditions as in Experiment 1, but with three density levels - the lowest, middle, and highest levels from Experiment 1. The subjects responded four times to each of the 12 conditions.

\section{Results}

The subjects placed the probe dot outside of the cylinder on 73\% of the trials (see Figure 7). An ANOVA of the CEs for the three density levels, two gaps, and two radii yielded a significant main effect for density $[F(2,10)=$ $8.86, p<.05, \omega^{2}=.188$ ]. As the texture-density level increased, subjects placed the probe dot farther outside the surface, with mean CEs of $-3.3,29.7$, and 44.0 for the three density levels. Post hoc comparisons (Tukey's $H S D$ test) showed the CE for the lowest density level to be significantly less than the CE for the middle and highest levels. There was a significant interaction between density and gap size $\left[F(2,10)=8.32, p<.01, \omega^{2}=.007\right]$, with the $C E$ increasing more rapidly between the lowest and middle density levels for the larger gap than for the smaller gap. The main effect of gap size was not significant. There were no trials on which the probe dot was placed outside the surface by more than 1.5 radii.

\section{Discussion}

Experiment 2 replicated the bias to place the probe dot outside the surface and the effect of density on that bias, indicating that these results were not due to the use of

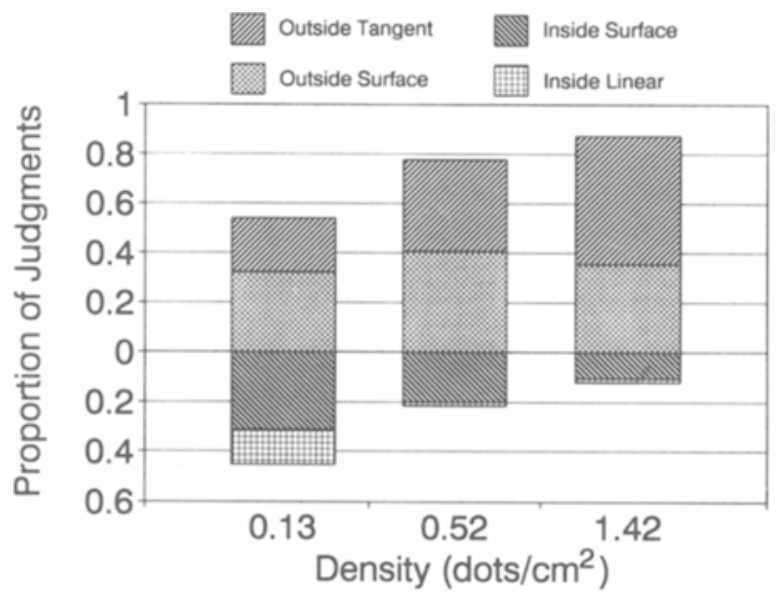

Figure 7. Probe-dot placement as a function of density in Experiment 2. uniform texture density on the surface of the cylinder in Experiment 1 (and the consequent texture gradient in the image). Use of a texture that is uniform in the projection results in a texture gradient that changes during rotation. Considering this disadvantage of beginning with a uniform 2-D texture, we conducted Experiments 3 and 4, like Experiment 1, with textures that were uniform on the surface of the cylinder.

\section{EXPERIMENT 3}

Experiments 3 and 4 examined in greater detail the effects of texture density and gap width on probe-dot placement. In particular, we were interested in the effects of these two variables on response variability. For this reason, we set more stringent criteria for subject selection that were intended to reduce overall variability, and we increased the number of repetitions of each condition. In preliminary experiments, we found that subjects who took less than $1 \mathrm{~min}$, on the average, to adjust the probe dot showed more variability in their responses than did subjects who responded more slowly. In Experiments 3 and 4 , we set a minimum average response time criterion and a maximum variability criterion (see Procedure). Because of the difficulty of the adjustment task, the length of time required to complete each adjustment, and the number of repetitions of each condition, we conducted separate studies of density and gap effects rather than combine the two variables in a larger factorial experiment. Experiment 3 included the five density levels examined in Experiment 1, as well as two additional levels with lower densities than those previously studied.

\section{Method}

The methods were the same as in Experiment 1, except as noted below.

Subjects. The subjects were 1 of the authors (A.S.), 1 graduate student, and 4 undergraduates. All of the undergraduates had participated in Experiments 1 and 2, but they had no knowledge of the purposes of the present experiment. They were paid for their participation.

Design. We examined one independent variable, texture density $\left(0.06,0.10,0.16,0.23,0.51,1.07\right.$, and $\left.1.43 \mathrm{dots} / \mathrm{cm}^{2}\right)$. The cylinder radius was 570 pixels (intermediate between the two radii used in Experiment 1), and the gap size was $54^{\circ}$. Each subject responded 10 times to each of the seven density conditions.

Procedure. Each subject participated in one practice session and two experimental sessions. The subjects were asked to participate in the experimental sessions if they met the following three criteria during the practice session: (1) average response time of at least $65 \mathrm{sec},(2)$ standard deviations ( $S D$ s) less than 35 pixels on at least three of the seven conditions, and (3) the probe dot not placed outside the surface by more than 1.5 times the radius on $10 \%$ or more of the trials. (All of the subjects met all three criteria.) Each session (practice and experimental) consisted of five randomized repetitions of the seven density conditions with a 5 -min break and 2 min of dark adaptation between the third and fourth repetition. The subjects were instructed as in Experiment 1, with additional emphasis on our concern with "accuracy and not with time."

\section{Results}

Overall, the subjects placed the probe dot outside the cylinder on $56 \%$ of the trials. This percentage varied 


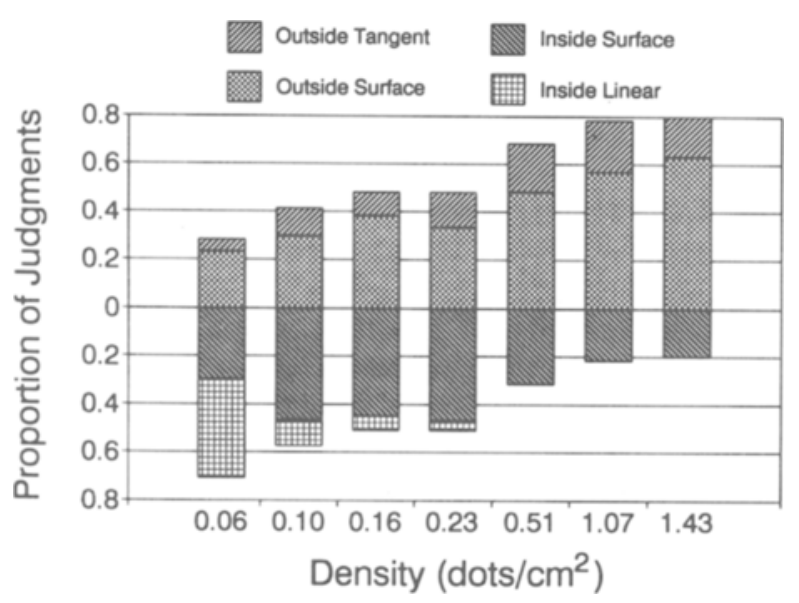

Figure 8. Probe-dot placement as a function of density in Experiment 3.

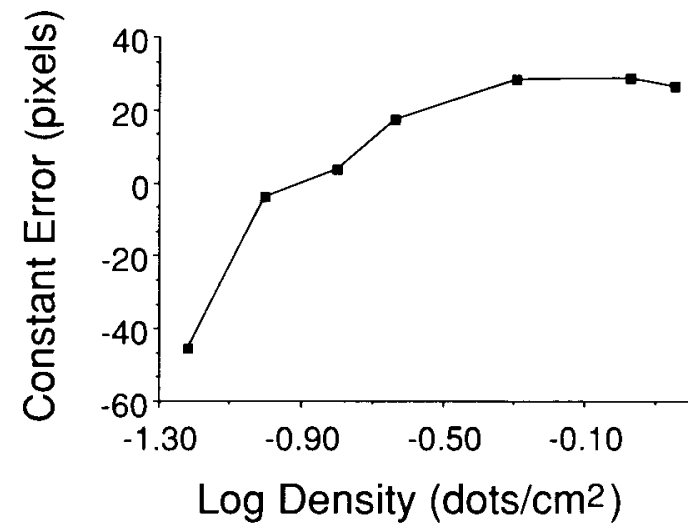

Figure 9. Constant error as a function of $\log$ density in Experiment 3.

between $27 \%$ at the lowest density level and $80 \%$ at the highest density level (see Figure 8). An ANOVA of the signed distance between the probe dot and the simulated surface for the seven density levels showed a significant main effect for density $\left[F(6,30)=13.02, p<.01, \omega^{2}=\right.$ $.281]$. The subjects placed the probe dot farther away from the surface at the higher density levels (see Figure 9). Post hoc comparisons (Tukey's HSD test) showed the CE for the lowest density level to be significantly less than the CEs for all other levels.

The SDs of the 10 responses made by each subject to stimuli at each of the seven density levels decreased significantly as density increased $[F(6,30)=3.79, p<.01$, $\left.\omega^{2}=.251\right]$. The mean $S D$ s for the seven density levels were $54.9,39.8,29.3,40.7,25.6,22.4$, and 20.0 pixels. All subjects reported seeing a 3-D object all the time and a cylinder most of the time.

\section{Discussion}

The $C E$ results in this experiment showed the same bias of placing the probe dot outside the surface as in Experiments 1 and 2. The bias increased with increasing den- sity, as in Experiments 1 and 2, although the bias was smaller at the lowest and two highest density levels. It is likely that the lower bias resulted from the increased experience of the undergraduates in the task, although no feedback was given in any of the experiments. The mean probe-dot position was between the surface and the tangent plane intersection for the five highest density levels. The $S D$ data show a high degree of precision in the responses at the higher density levels. For the three highest density levels, the mean probe-dot placement was more than one $S D$ outside the surface, indicating that the bias is reliable and not a consequence of response variability.

\section{EXPERIMENT 4}

Whereas the overall density of the texture defining the cylinder consistently affected probe-dot placement, Experiments 1 and 2 did not result in an effect of gap size. In Experiment 4, we examined this variable more closely by increasing the number of gap width levels to five and increasing the number of repetitions of each condition.

\section{Method}

The methods were the same as in Experiment 3, except as noted below.

Subjects. The subjects were five graduate students. Except for 1, A.S., they had no knowledge of the purposes of the experiment. Three undergraduates who had not participated in any of the previous experiments were run in practice sessions but failed to meet the selection criteria (see Procedure).

Design. We examined two independent variables, texture density $\left(0.53\right.$ pixels $/ \mathrm{cm}^{2}$ and 1.07 pixels $\left./ \mathrm{cm}^{2}\right)$ and gap size $\left(14^{\circ}, 29^{\circ}\right.$, $41^{\circ}, 54^{\circ}$, and $\left.83^{\circ}\right)$. The cylinder radius was the same as in Experiment 3 (570 pixels). Each subject responded 10 times to the 10 conditions.

Procedure. The subjects were asked to complete the two experimental sessions if they met two of the three criteria used in Experiment 3 . All 3 of the undergraduates failed to meet both the $S D$ and probe-dot placement criteria, and 2 of the 3 also failed to meet the response time criterion. Three of the graduate students failed to meet the time criterion in the practice trials (with mean response times of 49,52 , and $64 \mathrm{sec}$ ) but met the other two criteria. The practice session and the two experimental sessions each consisted of five blocks of the 10 stimulus conditions, randomly ordered within blocks, with a 5-min break and 2 min of dark adaptation between the third and fourth blocks.

\section{Results}

The subjects placed the probe dot outside the cylinder on $65 \%$ of the trials (see Figure 10). The mean CE across all conditions and subjects was 12.7 pixels. An ANOVA of the CE data yielded no significant main effects for either gap $[F(4,12)<1]$ or density $[F(1,3)<1]$, nor was the interaction significant. An ANOVA of the $S D$ s found a significant main effect for gap $[F(4,16)=12.54, p<.01$, $\left.\omega^{2}=.405\right]$. Post hoc comparisons (Tukey's $H S D$ test) showed the $S D$ for the largest gap width to be significantly greater than the $S D$ s for the other four gap widths. There was a significant interaction between gap and density $\left[F(4,16)=5.46, p<.01, \omega^{2}=.034\right]$ (see Figure 11). The main effect of density was not significant $[F(1,4)=$ $4.19, p>.05]$. 


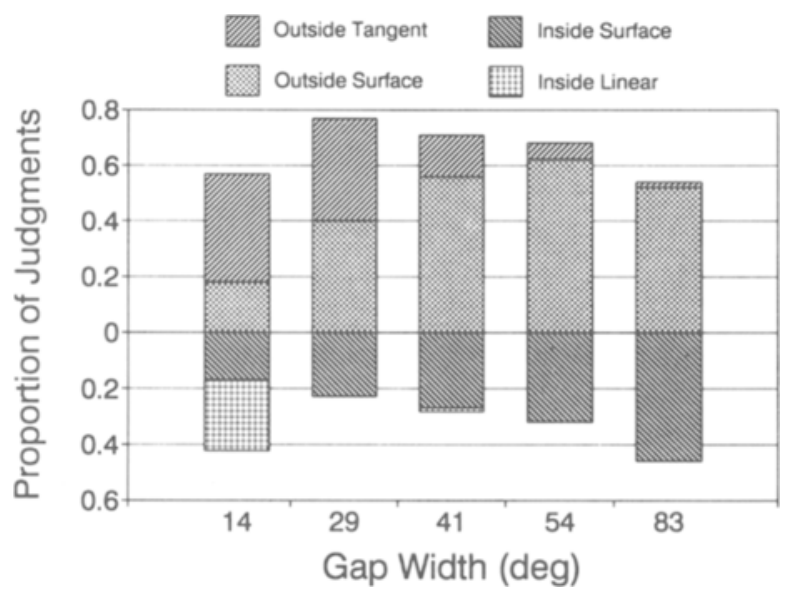

Figure 10. Probe-dot placement as a function of gap width in Experiment 4.

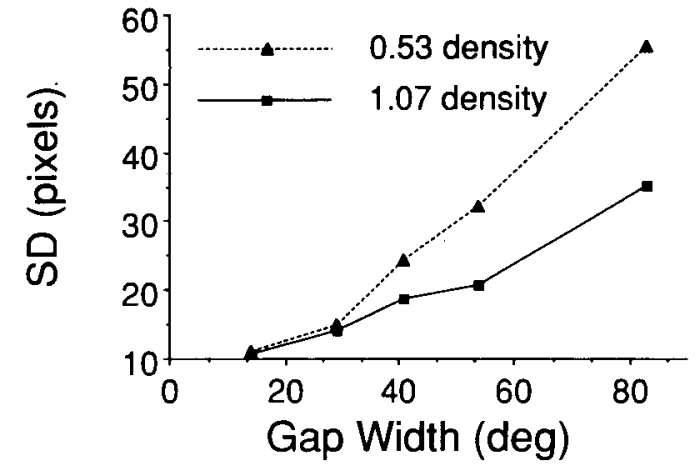

Figure 11. Standard deviation as a function of gap width in Experiment 4.

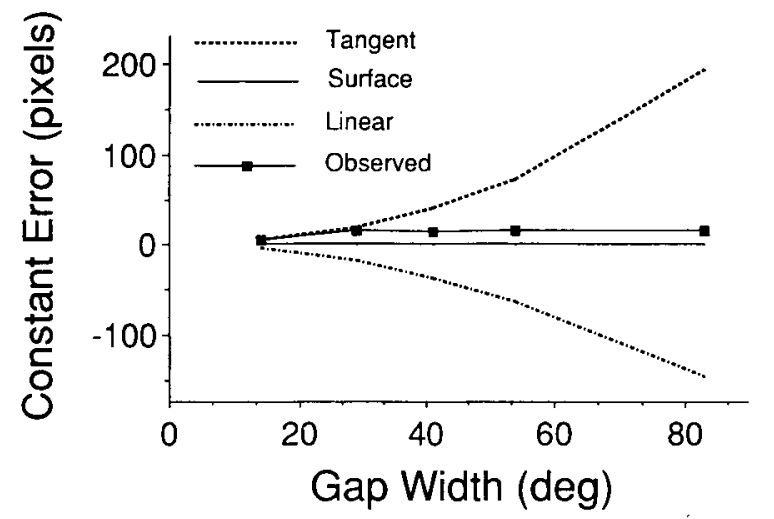

Figure 12. Constant error as a function of gap width in Experiment 4.

\section{Discussion}

Although both the linear interpolation and the tangent plane interpolation positions vary systematically with gap width, as shown in Figure 12, gap width does not have a significant effect on the $\mathrm{CE}$, which remains relatively constant across gap widths. This suggests that the bias in probe-dot placement reflects a constant error relative to the surface location and not a tendency to move the response toward the intersection of the tangent planes.

\section{EXPERIMENT 5}

The simulated objects in Experiments 1-4 had zero curvature in the vertical direction and constant curvature in the horizontal dimension. It is possible that the bias found in interpolation in these experiments might be a general effect of interpolating along an arc of constant curvature. To examine this possibility, we introduced a static, 2-D interpolation task in which subjects were asked to place a probe dot along a gap in a semicircular arc with the same radius as that of the cylinders studied in Experiments 3 and 4. A second purpose of Experiment 5 was to compare the $S D$ s found in the SFM task in Experiment 4 for varying gap widths with the $S D$ s for a static, 2-D interpolation task.

\section{Method}

Subjects. The subjects were 3 graduate students (A.S., A.M., P.G.) who were paid for their participation. Except for A.S., the subjects had no knowledge of the purposes of the present experiment. All three subjects had participated in Experiment 4.

Stimuli. The stimulus displays consisted of stationary semicircles with a radius of 570 pixels, equivalent to a top view of the cylinder. The semicircle was clipped in the $y$ dimension 21 pixels from the origin to make it comparable to the cylinder displays. The texture density was based on a top view of the 0.51 pixels $/ \mathrm{cm}^{2}$ condition from Experiment 4, yielding approximately $5.15 \mathrm{dots} / \mathrm{cm}$ along the contour. A gap was located in the center of the semicircular arc, and a probe dot was positioned in the gap along a radius intersecting the center of the gap (see Figure 13). The initial position of the probe dot varied randomly between 50 and 200 pixels from the curve (or more precisely, from the point at which a radius drawn through the probe dot would intersect the semicircle if the semicircle were completed through the gap).

Subjects used a joystick to move the middle dot vertically along a radius of the semicircle. The range of adjustment of the probe dot was 98 to 1638 pixels from the center of the semicircle. Forward motion of the joystick moved the middle dot inside the semicircle, and backward motion of the joystick moved the middle dot outside the semicircle.

Design. We examined one independent variable: gap size $\left(14^{\circ}\right.$, $29^{\circ}, 41^{\circ}, 54^{\circ}$, and $83^{\circ}$ ). Each subject responded 10 times to the five gap conditions.

Apparatus. The graphics display, viewing apparatus, and response device were the same as in the previous experiments. The semicircle radius was 570 pixels $\left(6.23^{\circ}\right)$. The position of the probe dot

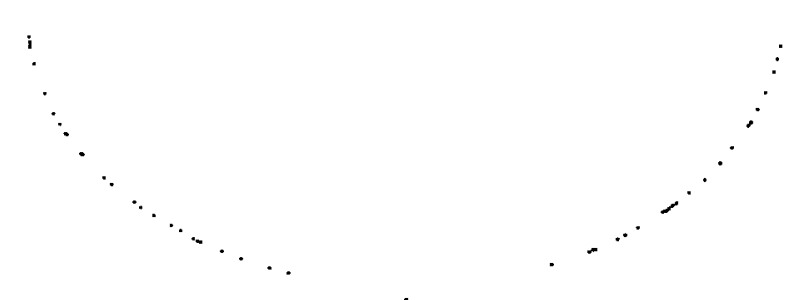

Figure 13. Example of a static two-dimensional stimulus used in Experiment 5. (The actual displays were white dots on a black background.) 


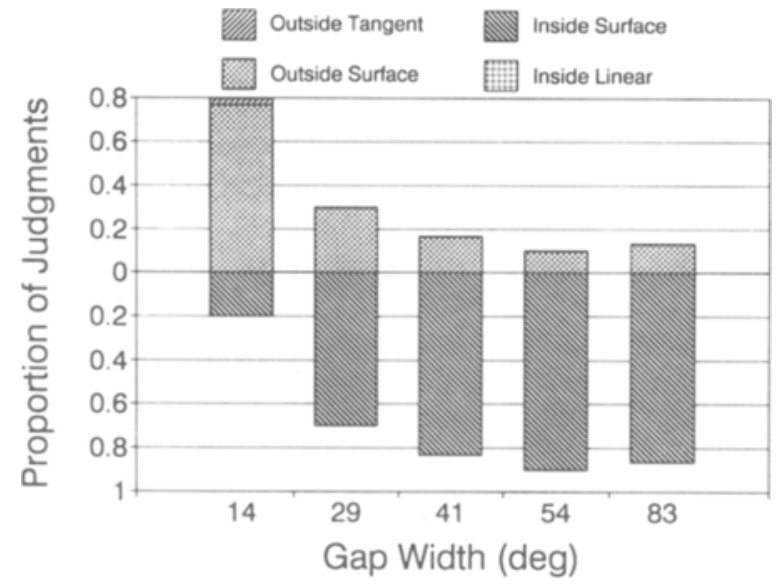

Figure 14. Probe-dot placement as a function of gap width in Experiment 5.

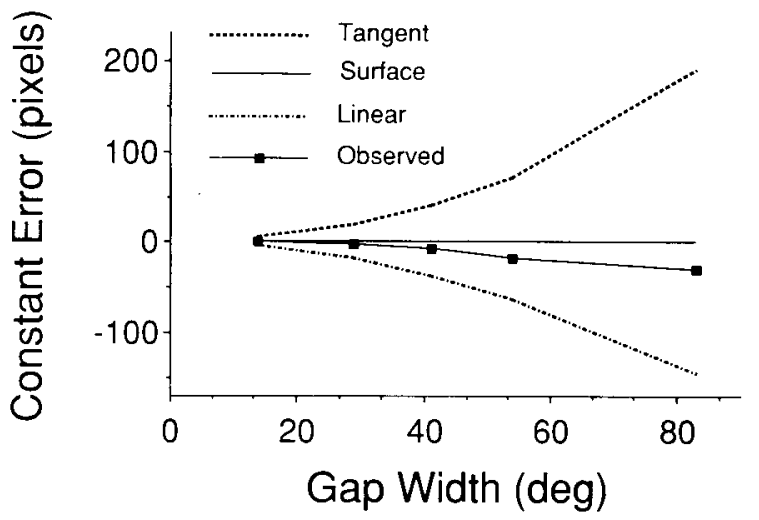

Figure 15. Constant error as a function of gap width in Experiment 5 .

along the $y$-axis was recorded by the computer when the button was pressed.

Procedure. Each subject participated in one practice session and one experimental session. The practice session consisted of five randomized repetitions of the five gap conditions. The criteria for including a subject were the same as in Experiment 4, and they were met by all subjects. The experimental session consisted of two blocks of five randomized repetitions of the five gap conditions, with a 5 -min rest period and $2 \mathrm{~min}$ of dark adaptation between the two blocks. The subjects were told that the displays of light dots on a dark background represented "a continuous curve with a missing middle part" and were instructed to "move the middle dot until it is on the unseen portion of the curve."

\section{Results}

The subjects placed the probe dot outside the semicircle on $30 \%$ of the trials (see Figure 14). An ANOVA of the signed distance between the probe dot and the simulated curvature for the five gap widths revealed a significant main effect for gap width $\left[F(4,8)=6.84, p<.05, \omega^{2}=\right.$ $.49]$. As the gap width increased, the probe dot was placed farther inside the semicircle and thus closer to a line connecting the endpoints of the gap (see Figure 15). Post hoc comparisons (Tukey's $H S D$ test) showed significant differences between the $14^{\circ}$ gap and the $41^{\circ}, 54^{\circ}$, and $83^{\circ}$ gaps, as well as between the $29^{\circ}$ gap and the $83^{\circ}$ gap.

An ANOVA of the $S D$ s revealed a significant main effect for gap $\left[F(4,8)=10.28, p<.01, \omega^{2}=.73\right]$, with $S D$ increasing with increases in gap width. The $S D$ s for the five gap widths were $11.1,8.1,4.1,2.7$, and 1.4 pixels. Post hoc comparisons (Tukey's HSD test) showed significant differences between the $14^{\circ}$ gap and the $54^{\circ}$ and $83^{\circ}$ gaps and between the $29^{\circ}$ gap and the $83^{\circ}$ gap.

\section{Discussion}

These results indicate that the bias found in probe-dot placement in the SFM task is not a general characteristic of curvature interpolation. Bias in the 2-D static task, with the same curvature and gap sizes, was in a direction opposite that in the 3-D task. The magnitude of the CE in probe-dot placement, on the other hand, was similar in the two tasks. The mean CE in probe-dot placement for the five gap levels ranged from +1.38 pixels to +19.23 pixels for the SFM displays in Experiment 4 and from +1.01 pixels to -29.69 pixels for the 2-D displays in Experiment 5. For the SFM displays, these CEs represent velocity differences of $0.12^{\prime} / \mathrm{sec}$ to $1.66^{\prime} / \mathrm{sec}$ and differences in the extent of the 2-D projected motion of $0.15^{\prime}$ to 2.06 '. For the 2-D displays, these represent projected differences of $0.33^{\prime}$ to 9.76 '. Variability was greater for the SFM displays. The $S D$ s across the five gap widths varied between 10.8 and 45.3 pixels for the SFM displays and between 1.4 and 11.1 pixels for the 2-D static displays.

\section{GENERAL DISCUSSION}

Subjects observing SFM displays can reliably judge the 3-D location of a simulated surface, even in regions devoid of feature points. At the same time, they show a consistent bias in these judgments. The reliability of interpolation judgments, as measured by the $S D$, is affected both by the texture density in the textured region of the surface and by the size of the region in which texture is absent. The $S D$ increases as (1) the gap width increases, or (2) the texture density decreases. These findings are not surprising: If the visible features constrain the interpolation process, the reliability of this process should increase as (1) distance between these features and the probe dot decreases, or (2) the density of these features on the surface increases. Perhaps more interesting is the level of reliability that subjects achieved. For the smallest gap and largest density studied in Experiment 4, the mean $S D$ across subjects was 10.5 pixels or $1.8 \%$ of the radius of the cylinder. These SDs can be compared to those obtained in two control experiments. The subject in both control experiments was our most experienced subject, A.S. In both experiments, the stimulus was, as before, a textured cylinder with a central gap. Now, however, one additional dot was placed on the cylinder in the center of the gap. Let us call this dot "D." In the first control experiment, a probe dot, under joystick control, could be moved back 
and forth along the line passing through $\mathrm{D}$ and passing, perpendicularly, through the major axis of the cylinder. The subject's task was to position the probe dot along this line so that it was coincident with $\mathrm{D}$. In the second control experiment, the probe dot could be moved, not along the line just mentioned, but along a line just below and parallel to it. The subject's task was to position the probe dot along this line so that the probe dot was aligned vertically with $D$. In the first control experiment, the mean $S D$ across seven blocks of trials was 7.47 pixels, just slightly below the lowest value found in the interpolation experiments. This indicates that reliability in the task of placing a probe dot on a simulated surface is almost as high as reliability in the task of simply keeping two dots coincident. The mean $S D$ in the second task-namely, the task of aligning two dots vertically-was 1.54 pixels, demonstrating that even higher reliability can be achieved in an alignment task than in the interpolation task or in the task of keeping two dots coincident.

The reliability of probe-dot placement in the interpolation experiments confirms the usefulness of the SFM probe. Under 2-D projection, an SFM probe is a dot that moves back and forth in phase with the motion of the dots on the simulated surface. The motion of the probe dot and the surface dots is always consistent with a single rigid 3-D configuration. The subject uses a joystick to vary the amplitude of the motion function of the SFM probe dot, while always leaving its phase unchanged. The small $S D s$ obtained in the high-density and small-gap conditions, over trials with different random distributions of surface dots, indicate that subjects can reliably use an SFM probe to indicate the location of a perceived surface.

Although interpolation was highly reliable, it was consistently biased. The obtained bias can be evaluated with respect to the three interpolation schemes that we discussed earlier (following Grimson, 1981): (1) linear interpolation between the depth simulated at the edges of the gap, or, equivalently, between the velocities of the dots at the edges of the gap; (2) interpolation of the simulated cylindrical surface as continuing through the gap with constant curvature; and (3) interpolation based on the intersection of planes tangent to the surface at the edges of the gap. Our finding that, under most conditions, the probe dot was placed outside the cylinder's surface might at first seem consistent with a compromise between Scheme 2 and Scheme 3. This possibility, however, is contradicted by the results obtained when gap width was varied: Although the location of the tangent plane intersection (Scheme 3) varied with gap width, the CE did not vary with gap width, but remained a constant proportion of the cylinder radius.

Our CE results-specifically the bias to place the probe dot outside the cylinder-are significant for theoretical accounts of surface reconstruction. Recall, for instance, the quadratic variation functional discussed in the introduction. Grimson (1981) investigated this functional, in part, because it is conservative: the interpolating surfaces it yields do not have extraneous inflections of curvature between visible features; they are "smooth" interpolations. Our results appear to be inconsistent with interpolation of a surface through the simulated feature points using this functional, or using functionals based on similar conservative assumptions, such as the square Laplacian functional. The interpolating surfaces that optimize these functionals do not pass outside the cylinder in the manner indicated by our subjects. There are several possible reasons why our results appear to be inconsistent with these theoretical accounts of surface interpolation. First, subjects might have misperceived the depths of the visible feature points. There is certainly evidence that depth can be misperceived in SFM displays. Most often, however, the misperceived depth is less than the simulated depth (Loomis \& Eby, 1988; Proffitt, Rock, Hecht, \& Shubert, in press) and thus would not account for our results. Although some studies have found perceived depth in SFM displays to exceed the simulated depth (Tittle, Braunstein, \& Liter, 1990; Todd \& Norman, 1991), judgments that we have obtained with SFM displays of cylinders (Braunstein, Tittle, \& Myers, 1988) indicate that cylinders displayed through SFM appear flattened. Another possibility is that subjects accurately interpolated a cylinder through the gap but were unable to place the probe dot accurately on the perceived cylinder. This seems unlikely, given that (1) the $S D$ was smaller than the CE in a number of conditions and (2) the CE was less than one half pixel in the two control experiments, mentioned above, that did not require interpolation. (The mean CEs in the control experiments were +0.24 and -0.47 pixels, respectively.) Since these alternative explanations of our results appear unlikely, one possibility to be considered is that subjects do not interpolate, in the sense of requiring the surface to pass through the visible feature points, but instead "reconstruct" - that is, fit a surface to the data that passes near, but not necessarily through-the positions of the feature points.

The results were quite different for static, 2-D interpolation using arcs of circles (having the same curvature and gap widths as were used with the cylinders). For the smallest gap width, the $S D$ of probe-dot placements (1.40 pixels) was much less than that found for 3-D interpolation and was comparable to that found in the second control experiment, using a dot-alignment task. The CE was in a direction opposite to that found in 3-D surface interpolation. Moreover, the $\mathrm{CE}$ increased in magnitude with gap width. It did so in a manner suggesting a compromise between (1) placing the probe dot on the unseen portion of the curve and (2) placing it on the line that connects the visible edges of the curve.

Several important questions remain:

1. Do the reliability and bias that we found for SFM interpolation apply to stereo interpolation stimuli of the type studied by Grimson (1981)? Although Grimson pro- 
vides some descriptive results, quantitative data on stereo interpolation across gaps in smoothly curved surfaces are not available to resolve this issue.

2. Do our findings with cylinders apply to more complex shapes?

3. How does the interpolation process handle discontinuities in curvature? This is an especially important issue for determining the relevance of various theoretical accounts of interpolation to human vision.

In summary, we have found that subjects can, with little variability, place an SFM probe dot on a surface, even in regions in which there are no visible feature points (except when the texture density elsewhere on the surface is very low). At the same time, subjects have a clear bias toward placing the SFM probe dot outside the surface. These results constrain theoretical accounts of how human observers might reconstruct surfaces between visible feature points. They also indicate that, in any SFM display in which points are used to represent a surface, the shape of that surface as simulated by the experimenter might differ from the shape of that surface as perceived by a subject, particularly in regions between visible feature points.

\section{REFERENCES}

Blake, A. (1984). Reconstructing a visible surface. In Proceedings of AAAI Conference 1984 (pp. 362-365). Los Altos, CA: AAAI Press.

Blake, A., Zisserman, A. (1986). Invariant surface reconstruction using weak continuity constraints. In Proceedings of Computer Vision and Pattern Recognition (pp. 62-67). Washington, DC: IEEE Computer Society Press.

Blake, A., Zisserman, A. (1987). Visual reconstruction. Cambridge, MA: MIT Press.

BRADY, M., \& HoRN, B. K. P. (1981). Rotationally symmetric operators for surface interpolation (Memo No. 654). Cambridge, MA: MIT Artificial Intelligence Laboratory.

Braunstein, M. L. (1962). Depth perception in rotating dot patterns: Effects of numerosity and perspective. Journal of Experimental Psychology, 64, 415-420.

Braunstein, M. L. (1976). Depth perception through motion. New York: Academic Press.

Braunstein, M. L., \& Andersen, G. J. (1984). Shape and depth perception from parallel projections of three-dimensional motion. Journal of Experimental Psychology: Human Perception \& Performance, $10,749-760$.

Braunstein, M. L., Hoffman, D. D., \& Pollick, F. E. (1990). Discriminating rigid from nonrigid motion: Minimum points and views. Perception \& Psychophysics, 47, 205-214.

Braunstein, M. L., Hoffman, D. D., Shapiro, L. R., Andersen, G. J., \& BENNETT, B. M. (1987). Minimum points and views for the recovery of three-dimensional structure. Joumal of Experimental Psychology: Human Perception \& Performance, 13, 335-343.

Braunstein, M. L., TitTle, J. S., \& Myers, T. S. (1988). Size constancy in orthographic structure-from-motion projections. Investigative Ophthalmology \& Visual Science, 29(3, Suppl.), 249.

Braunstein, M. L., \& TodD, J. T. (1990). On the distinction between artifacts and information. Joumal of Experimental Psychology: Human Perception \& Performance, 16, 211-216.

Brookes, A., \& Stevens, K. A. (1989). Binocular depth from surfaces versus volumes. Journal of Experimental Psychology: Human Perception \& Performance, 15, 479-484.

BülthofF, H. H., MALLOT, H. A. (1987). Interaction of different modules in depth perception. Investigative Ophthalmology \& Visual Science, 27(3, Suppl.), 293.
Cornilleau-Pérès, V., Droulez, J. (1989). Visual perception of surface curvature: Psychophysics of curvature detection induced by motion parallax. Perception \& Psychophysics, 46, 351-364.

Doner, J., Lappin, J. S., \& Perfetto, G. (1984). Detection of threedimensional structure in moving optical patterns. Joumal of Experimental Psychology: Human Perception \& Performance, 10, 1-11.

DOSHER, B. A., LANDY, M. S., \& SPERLing, G. (1989). Ratings of kinetic depth in multidot displays. Journal of Experimental Psychology: Human Perception \& Performance, 15, 816-825.

GREEN, B. F., JR. (1961). Figure coherence in the kinetic depth effect. Joumal of Experimental Psychology, 62, 272-282.

Grimson, W. E. L. (1981). From images to surfaces: A computational study of the human early visual system. Cambridge, MA: MIT Press.

JULESz, B. (1971). Foundations of cyclopean perception. Chicago: University of Chicago Press.

Julesz, B., \& Frisby, P. (1975). Some new subjective contours in random-line stereograms. Perception, 4, 145-150.

LapPIN, J. S., Doner, J. F., \&otTas, B. (1980). Minimal conditions for the visual detection of structure and motion in three dimensions. Science, 209, 717-719.

LOOMIS, J. M., \& EBY, D. W. (1988). Perceiving structure from motion: Failure of shape constancy. In Proceedings of the Second International Conference on Computer Vision (pp. 383-391). Washington, DC: IEEE Computer Society Press.

MARROQUIN, J. (1985). Probabilistic solution of inverse problems. Unpublished doctoral dissertation, Massachusetts Institute of Technology.

McLauchlan, P., Zisserman, A., \& Blake, A. (1987). Knowledge source for describing stereoscopically viewed textured surfaces. Image \& Vision Computing, 5, 105-110.

Norman, J. F., LAPPIN, J. S. (1991). The perception of curved surfaces defined by optical motion. Manuscript submitted for publication.

Petersik, J. T. (1987). Recovery of structure from motion: Implications for a performance theory based on the structure-from-motion theorem. Perception \& Psychophysics, 42, 355-364.

Proffit, D. R., Rock, I., Hecht, H., \& ShUbert, J. (in press). The stereokinetic effect and its relation to the kinetic depth effect. Journal of Experimental Psychology: Human Perception \& Performance.

Sperling, G., Landy, M. S., Dosher, B. A., \& Perkins, M. E. (1989). The kinetic depth effect and identification of shape. Journal of Experimental Psychology: Human Perception \& Performance, 15, 816-825.

STEVENs, K. A. (1981). The visual interpretation of surface contours. Artificial Intelligence, 217, 47-74.

STEVENS, K. A. (1983). The line of curvature constraint and the interpretation of 3-D shape from parallel surface contours. In Eighth International Joint Conference on Artificial Intelligence (pp. 1057-1061). Los Altos, CA: William Kaufmann.

SteVENS, K. A. (1986). Inferring shape from contours across surfaces. In A. P. Pentland (Ed.), From pixels to predicates: Recent advances in computational vision (pp. 93-110). Norwood: NJ: Ablex.

Stevens, K. A., B Brookes, A. (1987). Probing depth in monocular images. Biological Cybernetics, 56, 355-366.

Terzopoulos, D. (1984). Multi-level computation of visual surface representation. Unpublished doctoral dissertation, Massachusetts Institute of Technology.

Terzopoulos, D. (1986). Integrating visual information from multiple sources. In A. P. Pentland (Ed.), From pixels to predicates: Recent advances in computational vision (pp. 111-142). Norwood, NJ: Ablex.

Tittle, J. S., Braunstein, M. L., \& Liter, J. C. (1990). Recovering surface slant from orthographic projections. Investigative Ophthalmology \& Visual Science, 31, 523.

TODD, J. T., AKerstrom, R. A., REICHEL, F. D., \& HAYES, W. (1988). Apparent rotation in three-dimensional space: Effects of temporal, spatial, and structural factors. Perception \& Psychophysics, 43, 179-188.

ToDD, J. T., \& Norman, J. F. (1991). The visual perception of smoothly curved surfaces from minimal apparent motion sequences. Perception \& Psychophysics, 50, 509-523.

Treue, S., Husain, M., \& ANDERSEN, R. A. (1991). Human perception of structure from motion. Vision Research, 31, 59-75. 
UlLman, S. (1979). The interpretation of visual motion. Cambridge, MA: MIT Press.

Uttal, W. R., Davis, N. S., Welke, C., Kakarala, R. (1988) The reconstruction of static visual forms from sparse dotted samples. Perception \& Psychophysics, 43, 223-240.

Wallach, H., O'ConNell, D. N. (1953). The kinetic depth effect. Journal of Experimental Psychology, 45, 205-217.

WEISs, I. (1990). Shape reconstruction on a varying mesh. IEEE Transactions on Pattern Analysis and Machine Intelligence, 12, 345-362.

WürGER, S. M., LANDY, M. S. (1989). Depth interpolation with sparse disparity cues. Perception, 18, 39-54.

\section{NOTES}

1. In the present study, the curvature of the cylinder was always in the direction of rotation. This was necessary to avoid changes in texture density during rotation. Studies of curvature discrimination (ComilleauPérès \& Droulez, 1989; Norman \& Lappin, 1991) have found that the relationship between the direction of curvature and the axis of rotation affects accuracy in discriminating curved from flat surfaces.

2. The term pixel does not strictly apply to the calligraphic displays that were used in these experiments but is used here to refer to distinct plotting positions.

3. Errors were measured in pixel units along a diameter of the cylinder in simulated 3-D space. These are the same units as those used to describe the cylinder radius, and the errors can be converted to proportion of the radius by dividing by the appropriate radius ( 500 or 640 pixels in Experiment 1 and 570 pixels in all other experiments). Errors can also be expressed as differences in velocity between the probe-dot velocity that would place the dot on the simulated surface and the probe-dot velocity selected by the subject. An error of 1 pixel is equivalent to an average velocity difference of $5.17^{\prime \prime}$ of arc, based on a rotation of $19^{\circ}$ in $1.24 \mathrm{sec}$.

(Manuscript received May 20, 1991; revision accepted for publication September 12, 1991.) 